\title{
Mathematizing Niemeyer's architecture through parametric modeling: evaluating the parables of the Pampulha Church
}

\section{SIGRADI2018 TECHNOPOLITICAS \\ xxii congresso da sociedade iberoamericana de gráfica digital 22th conference of the iberoamerican society of digital graphics 07|08|09|novembro|2018 iau usp | são carlos | sp br}

\author{
Verner Max Liger de Mello Monteiro \\ Instituto Federal do Rio Grande do Norte e Universidade Federal do Rio Grande do Norte | \\ Brazil |verner.monteiro@ifrn.edu.br
}

\author{
José Rauryson Alves Bezerra \\ Instituto Federal do Rio Grande do Norte | Brazil | rauryson.alves@ifrn.edu.br
}

Paulo Roberto Paulino do Nascimento

Instituto Federal do Rio Grande do Norte | Brazil | paulorobertomate@gmail.com

\section{Erisvaldo Ramalho dos Santos Júnior}

Instituto Federal do Rio Grande do Norte | Brazil | uniorramalho155j@gmail.com

\begin{abstract}
This paper describes the mathematization process behind the parables of the Pampulha Church, one of the most iconic buildings designed by Oscar Niemeyer, in order to check how applicable was the use of analytic geometry in his architecture. To reach this, we factored the second degree equations presented on the building based on the parable height and width, then using parametric modeling to translate the formula into shape. As a result, the study intended to demonstrate how equations can be integrated into architecture, identifying how conic curves are being applied to the architectural geometry.
\end{abstract}

Keywords: Parametric modeling; Pampulha church; Oscar Niemeyer; Analytic geometry.

\section{INTRODUÇÃO}

A arquitetura de Oscar Niemeyer está iconicamente ligada à representação do espaço a partir de formas geométricas, usando frequentemente curvas cônicas. A igreja de São Francisco de Assis, também conhecida como igreja da Pampulha, está entre alguns dos projetos que dão início a um modo particular de conceber arquitetura para Oscar Niemeyer (Gonçalves, 2010). Nesse projeto, 0 arquiteto desafia as técnicas construtivas estabelecidas da época, investigando, juntamente com o engenheiro Joaquim Cardozo, a utilização da superfície como elemento de sustentação. Souza (2012) atesta a importância do uso da forma parabólica neste projeto como modo de um único elemento fazer o papel estrutural da cobertura e paredes. Considerando a falta de registros sobre critérios geométricos detalhados que foram utilizados para essa obra, o objetivo desse artigo é investigar quais fórmulas matemáticas das curvas parabólicas traduzem a forma da igreja, com o auxílio da modelagem paramétrica e prototipagem rápida, visando a matematização da volumetria.

Como resultado, nosso estudo teve o propósito de demonstrar como as curvas cônicas estão sendo aplicadas em formas arquitetônicas, e se estão obedecendo as características matemáticas descritas por equações.

\section{MATEMÁTICA, MODELAGEM PARAMÉTRICA E A ARQUITETURA DE NIEMEYER}

No início do século XX, Le Corbusier observou que, por mais nova ou diferente que a arquitetura moderna fosse, a matemática ainda estava em sua essência (Burry \& Burry, 2010).

Arquitetura e geometria têm relação desde a sua concepção até a sua materialização. Ambas tem o poder de expressar e organizar espaço usando conceitos fora de restrições e em busca da representação física (Burry \& Burry, 2010). A geometria busca generalizações, que uma vez estabelecidas (demonstradas e provadas), ficam disponíveis para serem utilizadas; arquitetura aplica essas relações genéricas construtivamente para criar relações espaciais específicas.

Com o advento da gráfica digital, a matemática pode ter aplicações que vão desde a conectividade entre ambientes (Shekhawat, 2017) até a inserção de fórmulas em algoritmos, por meio da modelagem paramétrica. Com ela, criam-se modelos geométricos parametricamente com o uso da programação computacional visual, a exemplo das aplicações de equações matemáticas a algoritmos demonstradas por Tedeschi (2014).

A modelagem paramétrica é baseada na associação de geometria, onde descrições matemáticas parametrizadas 
e associações entre pontos, curvas, superfícies e sólidos são possíveis (Stals, Elsen, \& Jancart, 2017). Na arquitetura, aparece por volta dos anos 2000 (Woodbury, 2010), tendo sido associada com a produção de arquitetura não convencional (Kolarevic, 2008) da era digital.

Apesar de Oscar Niemeyer não dispor das ferramentas computacionais no período da arquitetura moderna, ele aplica a matemática em suas obras, usando a geometria para dar caráter singular a seus projetos, criando a sua própria linguagem. Ela aparece com o uso das formas geométricas espaciais simples, como prismas e sólidos de revolução, e quando 0 arquiteto toma partido de curvas cônicas (aquelas que são obtidas a partir de seções em cones) na sua forma arquitetônica, como as parábolas, as hipérboles e as elipses.

Florio \& Veiga (2016) estudaram a geometria em edifícios curvilíneos de Oscar Niemeyer no Memorial da América Latina. Nele, constataram que a modelagem paramétrica possibilitou identificar importantes princípios geométricos adotados pelo arquiteto. Por terem incorporado também a modelagem geométrica, devido os edifícios estudados não possuírem curvas cônicas em sua volumetria, verificaram a definição da forma de elementos mais simples e a análise do espaço arquitetônico. Motivados pela aplicabilidade desse estudo, foi dada continuidade e a análise foi ampliada para uma outra ótica: a verificação de fórmulas matemáticas na arquitetura de Niemeyer.

\section{METODOLOGIA}

A metodologia aplicada no decorrer desta pesquisa foi dividida em quatro etapas sequenciais (Figura 1):

1) Análise das Parábolas Arquitetônicas (APA), tomando como ponto de partida as medidas de base e da altura das parábolas encontradas na obra da igreja da Pampulha;

2) Aplicação do método matemático de Equação Fatorada da Parábola (EFP), para encontrar as fórmulas do percurso parabólico de cada uma das parábolas que aparecem na forma arquitetônica;

3) Modelagem Paramétrica (MP) das cascas de concreto que tem formas parabólicas. Essas equações foram introduzidas no software Rhinoceros e seu plug-in Grasshopper como dados de entrada em um algoritmo leitor de expressão matemática denominado Evaluate Surface (EV.SRF.). Depois, a equação foi transformada em pontos e em seguida em curvas. A partir delas, são realizadas uma série de Operações Geométricas Euclidianas (OGE), como rotação, translação e extrusão, criando a nave da igreja. Por último, Operações Geométricas Booleanas (OGB) de subtração de sólidos foram aplicados para criar as "parábolas" da parte posterior da edificação;

4) Análise Comparativa de Geometrias (ACG) para verificar o resultado encontrado para cada parábola do projeto. Posteriormente, foi gerado o modelo geométrico completo, por meio de algoritmos de extrusão, translação, cópia e operações matemáticas booleanas, comparando-as com a original da obra arquitetônica.

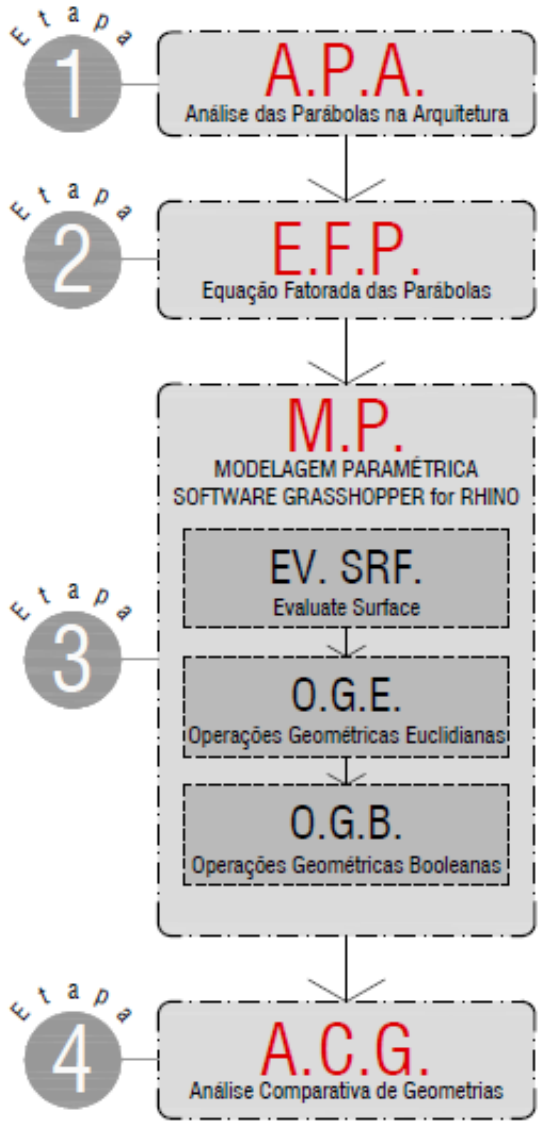

Figura 1 - Metodologia aplicada na pesquisa. Fonte: autores.

\section{ANÁLISE DAS PARÁBOLAS NA ARQUITETURA (APA)}

Curvas cônicas (Figura 2) são resultantes de seções no cone reto circular, que é aquele cuja base é uma circunferência e a projeção do vértice sobre o plano é o centro da circunferência, de acordo com lezzi (1993).
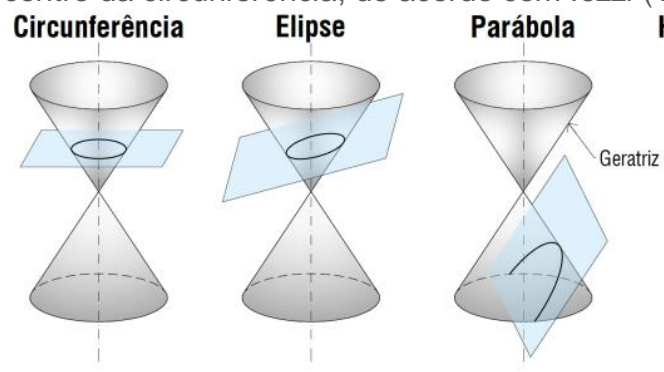

Hipérbole

Figura 2 - Processo de obtenção das curvas cônicas. Fonte: autores.

Ainda de acordo com o autor, a parábola trata-se de um conjunto de pontos de um plano que são equidistantes de uma reta diretriz e de um ponto focal, em um intervalo chamado domínio (Figura 3).

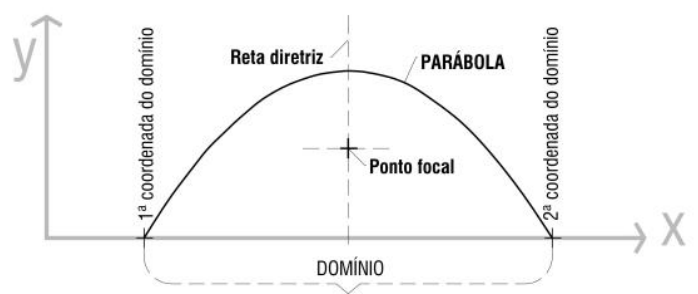

Figura 3 - Elementos de uma parábola. Fonte: autores.

$\mathrm{Na}$ Igreja da Pampulha, as parábolas aparecem como geradores primários da forma, que são extrudados para 
gerar as cascas de concreto armado. Identificamos cinco parábolas diferentes. Com base nos dados trazidos por (Pereira, 2012), nomeamos as parábolas para análise. As parábolas 1 e 2 são as maiores, e criam a nave da igreja e criam também uma segunda casca de concreto, que conecta-se às demais parábolas para cobrir o altar e a sacristia (Figura 4).
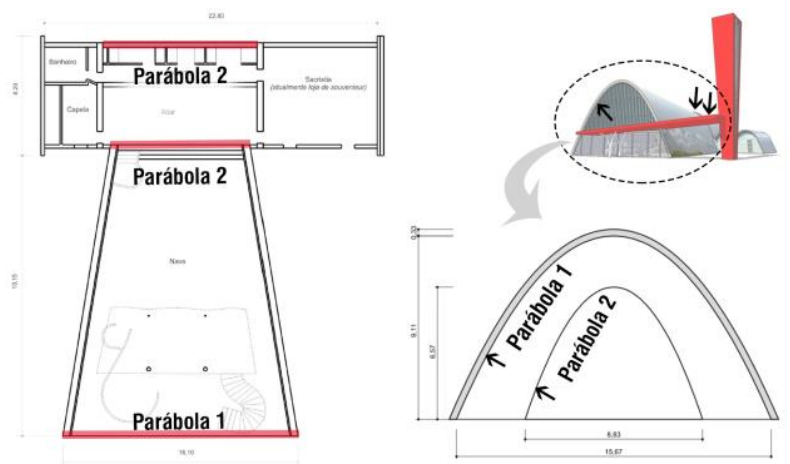

Figura 4 - Parábolas 1 e 2. Fonte: Adaptado de Pereira (2012).

Já as parábolas 3, 4 e 5 (Figura 5) possuem, a princípio, mesmas medidas de base e altura, diferenciando-se apenas pelo posicionamento e domínio. Esse posicionamento foi checado, em momento posterior, com os domínios das parábolas, na etapa de EFP.

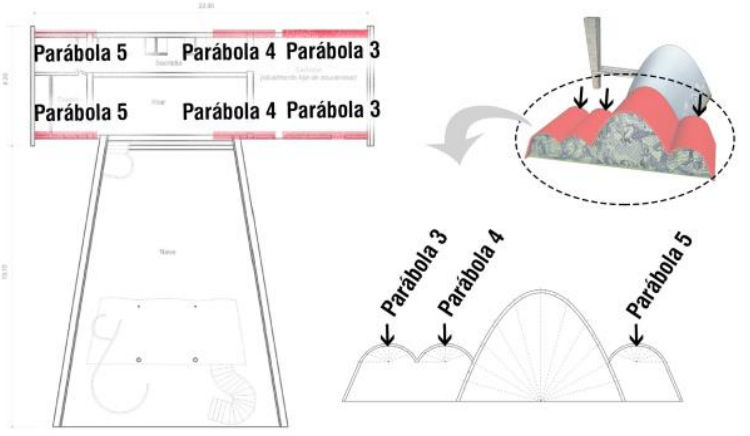

Figura 5 - Parábolas 3, 4 e 5. Fonte: Adaptado de Pereira (2012).

A forma dessas parábolas aparenta não respeitar características matemáticas da parábola, aproximando-se de um arranjo geométrico de arcos e segmentos de reta. $\mathrm{Na} 4^{\underline{a}}$ etapa desta metodologia, denominada ACG, será feita uma checagem e comparação com formas parabólicas para confirmar ou refutar essa característica.

\section{APLICACCÃO DO MÉTODO MATEMÁTICO DE EQUAÇẨO FATORADA DA PARÁBOLA (EFP)}

De uma forma geral podemos apresentar algebricamente pontos, retas ou cônicas no $\mathbb{R} \times \mathbb{R}$ por meio da equação:

$$
a x^{2}+b y^{2}+c x y+d x+e y+f=0
$$

com pelo menos um dos coeficientes $a, b, c, d$, e não nulo (Lima, Carvalho, Morgado, \& Wagner, 2007).

Por meio de manipulações na expressão dada é possível chegar a algumas equações reduzidas, que facilitam o reconhecimento da figura que será a representação geométrica no plano cartesiano.
No caso de uma parábola de vértice em (xo,yo), eixo de simetria vertical e parâmetro (distância entre o foco e o vértice) dado por $\mathrm{p}$, a expressão algébrica é dada por ( $\mathrm{x}$ $\left.x_{0}\right)^{2}=2 p\left(y-y_{0}\right)$. De forma análoga, se a parábola tem vértice em em (хо,уо), eixo de simetria horizontal e parâmetro $p$, a sua expressão algébrica é dada por ( $y$ yo $)^{2}=2 p\left(x-x_{0}\right)($ lezzi, 1993).

Para o desenvolvimento de cada equação das parábolas da obra de Niemeyer, foi necessário conhecer a dimensão da largura da base de cada parábola e da posição de um terceiro ponto qualquer. Achados esses pontos, foram inseridos na equação fatorada da parábola, que é da forma: $0=a\left(x-x^{\prime}\right) \cdot\left(x-x^{\prime \prime}\right)$ em que $x^{\prime}$ e x" são as raízes, ou soluções, da equação e "a" é um coeficiente da equação.

Parábola 1: Considerando um ponto genérico $P$, equidistante $\mathrm{R}$ unidades de $\mathrm{C}$, pode ser determinado pela equação:

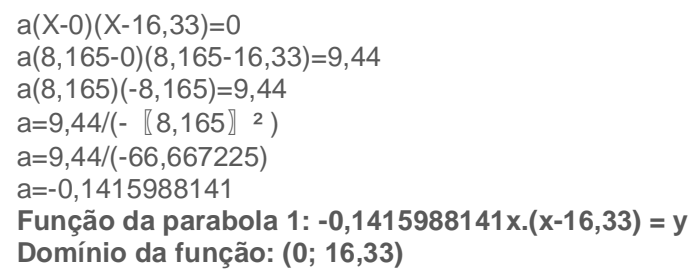

Parábola 2: Os pontos que definiram essa parábola, considerando o plano $\mathrm{xOy}$, sendo $x^{\prime}$ e $x$ " os pontos determinados pelas raízes tal que $x^{\prime}=\left(x^{\prime}, 0\right)$ e $x^{\prime \prime}=\left(x^{\prime \prime}, 0\right)$, teremos $x^{\prime}=(3,75 ; 0), x^{\prime \prime}=(12,58 ; 0)$ e vértice $(8,165$; $6,9)$.

$a(X-3,75)(X-12,58)=Y$

$\mathrm{a}(8,165-3,75)(8,165-12,58)=6,9$

$\mathrm{a}^{*} 4,415^{*}(-4,415)=6,9$

$a=6,9 /(-19,492225)$

$a=-0,3539872949$

Função da parabola 2: $-0,3539872949(X-3,75)(X-12,58)=Y$

Domínio da função: $(3,75 ; 12.58)$

Parábolas 3, 4 e 5: Os pontos que definem a equação de referência das parábolas da estrutura posterior, considerando $x O y$, teremos $x^{\prime}=(0 ; 0), x^{\prime \prime}=(3,673 ; 0) e$ vértice $(1,8365 ; 1,030)$.

$a(X)(X-3,673)=Y a(1,8365)(1,8365-3,673)=1,030$

$a^{*} 1,8365^{*}(-1,8365)=1,030$

$a=1,030 /(-3,37273225)$

$a=-0,3053903849$

Função referência das parábolas 3,4 e 5:

$-0,3053903849 X^{2}+1,1216988837 X=y$

Como os domínios das parábolas 3,4 e 5 são variáveis, utilizou-se apenas a equação da parábola 5 , e as demais foram obtidas por translação, descrito na etapa 3 como OGE, e intersecção de sólidos, descritos na etapa 4 como OGB.

Para posicionar parábola 5 no local correto, foi necessário movê-la a partir das dimensões de projeto, chegando, por fim, à seguinte equação e domínio:

$-0,3053903849(X-0,71)^{2}+1,1216988837(X-0,71)+2,71=y$ Domínio $(-0,954 ; 6,047)$ 


\section{MODELAGEM PARAMÉTRICA (MP)}

Com o uso do software Grasshopper for Rhino, o procedimento utilizado para fazer a modelagem paramétrica (MP) partiu da utilização das equações e domínios, encontrados na fase de EFP, para criar as curvas parabólicas de 1 a 5 por meio do procedimento Evaluate Surface (EV.SRF). A partir delas, foram adotadas uma sequência de Operações Geométricas Euclidianas (OGE) e Booleanas (OGB) (Figura 6).

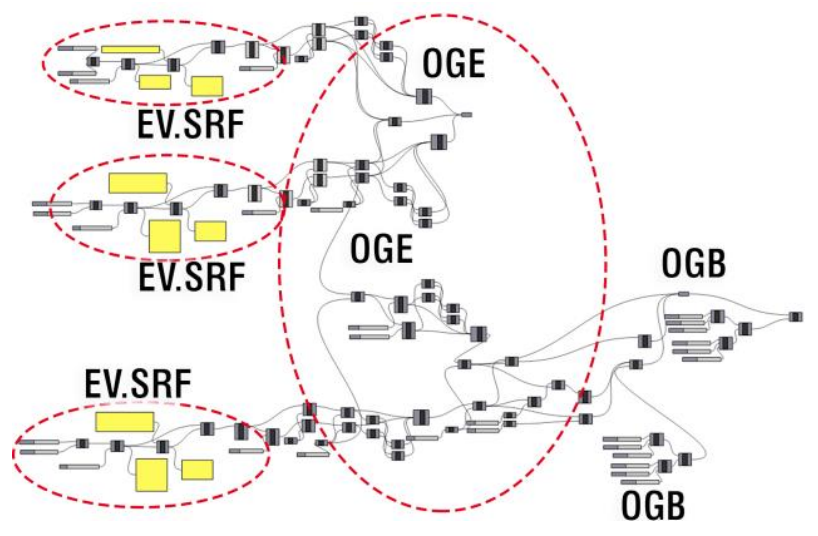

Figura 6 - Representação geral das operações por meio de algoritmos adotados no ambiente do Grasshopper. Fonte: autores.

\section{Evaluate Surface (EV.SRF)}

Cada equação encontrada na etapa anterior da metodologia foi inserida no componente Evaluate Surface, que combinado com os componentes Range e Construct Domain transforma equações em dados de saída. Esses dados viraram pontos em determinados intervalos da parábola, quando ligados ao componente Construct Point (Figura 7).

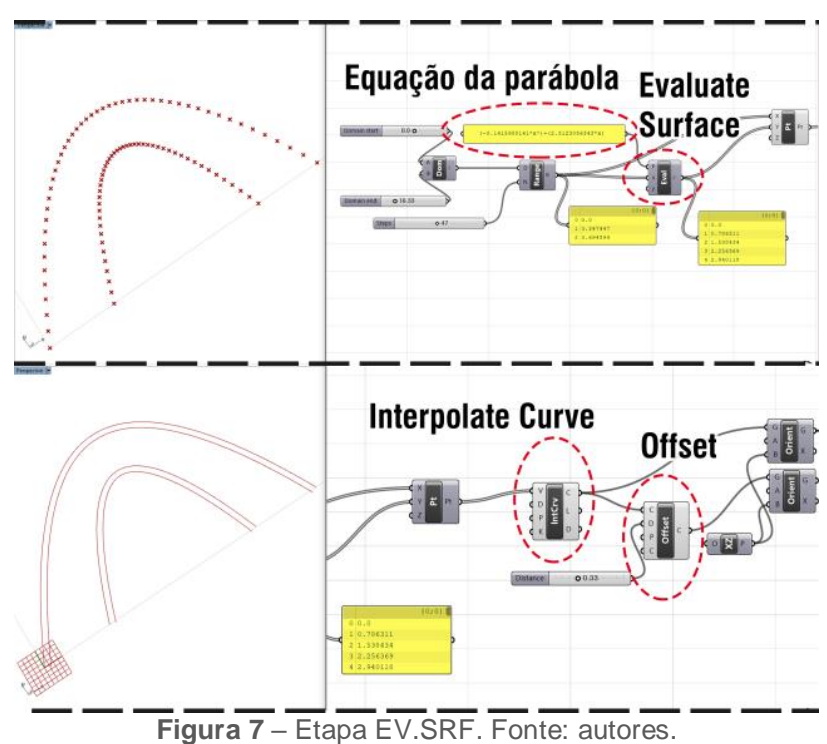

Operações Geométricas Euclidianas (OGE)

Nesta etapa, foram realizadas as seguintes Operações Geométricas Euclidianas para dar seguimento à modelagem paramétrica: rotação, translação e extrusão. Essa operação é executada através de uma sequência de componentes (Figura 8).
Para criar o espaço da nave da igreja, foi necessário, a partir das parábolas 1 e 2, criadas no plano XY, promover rotação com o componente Orient ao longo do eixo $X$, para deixa-las no plano $X Z$. Após isso, a parábola 2 foi translada com o componente move, gerando a profundidade da nave.

Para unir e criar a casca de concreto, foi utilizado o componente loft, que interpola superfícies considerando as curvas parabólicas 1 e 2 .

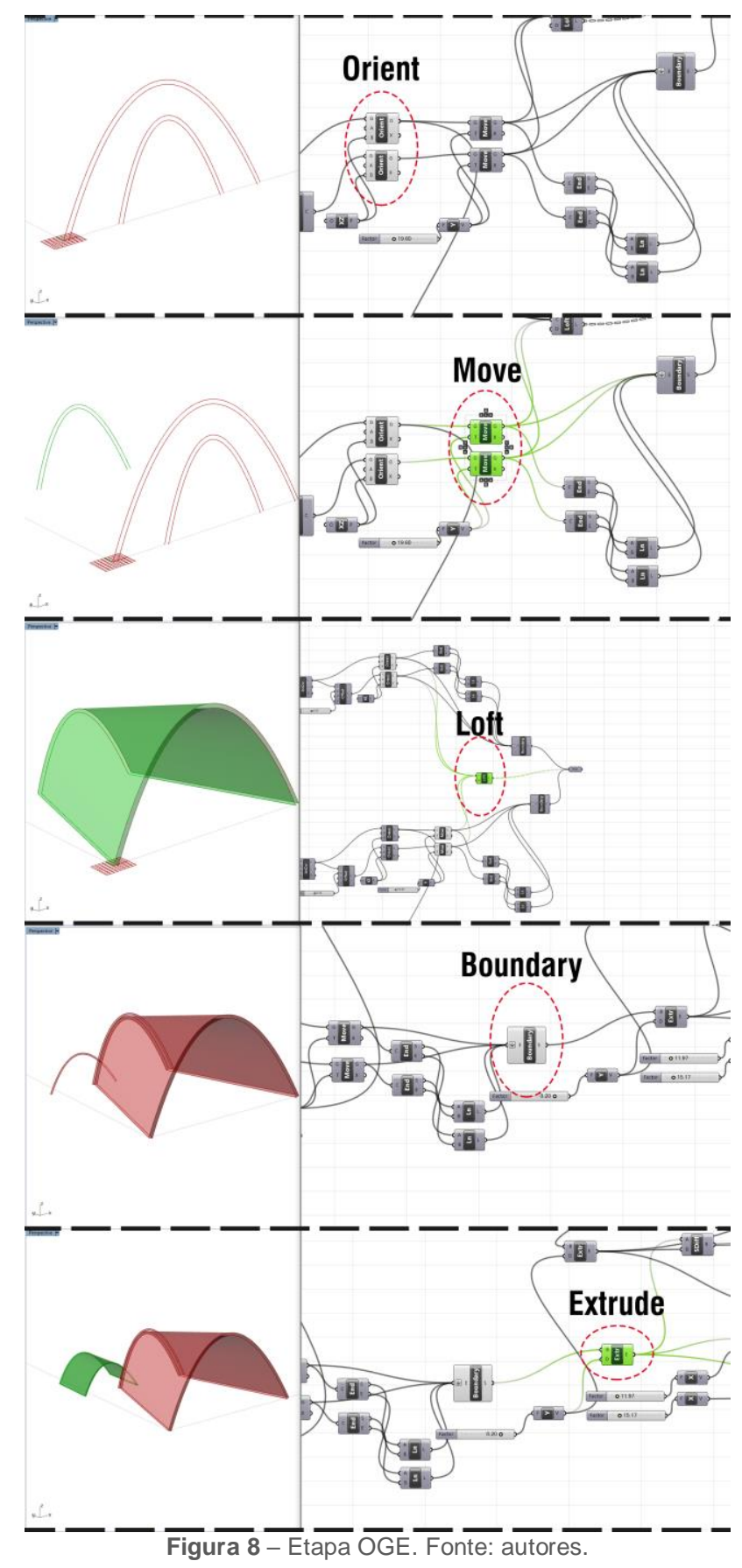

Em processo análogo ao das primeiras parábolas, a parábola 5 foi criada e translada para a posição que ocupa, e sua profundidade foi dada com o uso do componente extrude. Como etapa final da etapa OGE, foram aplicadas translações com o componente move (que move o objeto, mantendo o original que foi), dando origem às parábolas 3 e 4, mas ainda sem arremate dos seus encontros. 
Operações Geométricas Booleanas (OGB)

As operações Geométricas Booleanas utilizadas nesta última etapa da modelagem paramétrica foram uma sequência de subtrações de sólidos geométricos (Figura 9).

A primeira ação foi a criação da casca parabólica que cobre o altar e da sacristia, que são o prolongamento da nave da igreja. Apesar de ser uma Operação Geométrica Euclidiana, está nessa etapa por fazer parte da sequência de subtrações que foram realizadas. A partir daí, foi feita a subtração de sólido (Solid Difference) dessa forma em relação à casca de concreto originada da parábola 5 .

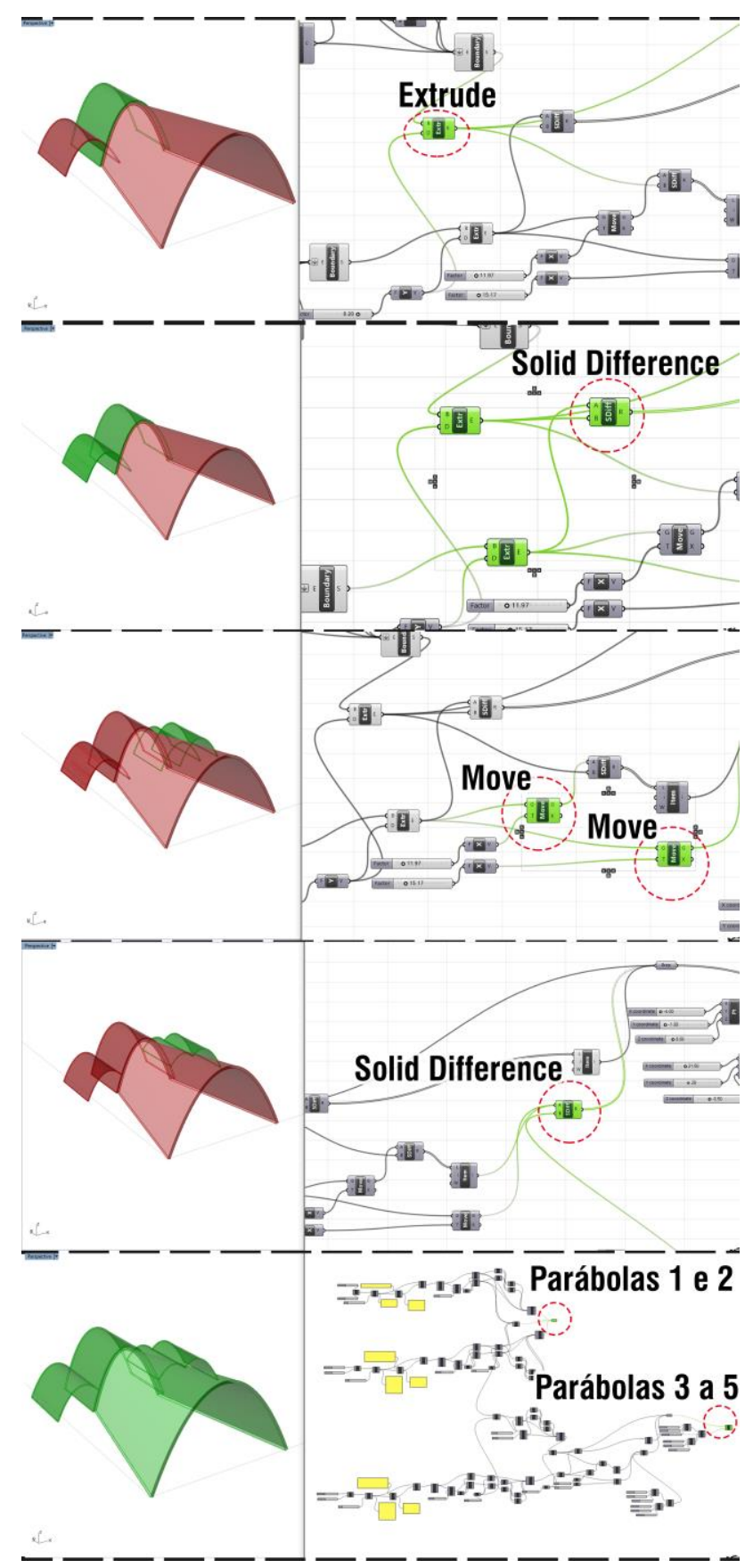

Figura 9 - Etapa OGB. Fonte: autores.

As etapas seguintes foram a translação da casca de concreto criada pela parábola 5 para dar origem às cascas de concreto que originaram das parábolas 3 e 4 .

\section{ANÁLISE COMPARATIVA DE GEOMETRIAS (ACG)}

Foram sobrepostas as parábolas 3, 4 e 5, em vermelho, comparando-as à geometria original da obra, em preto, no intuito de buscar distorções às formas parabólicas que foram obtidas a partir das equações desenvolvidas na etapa EFP (Figura 10).

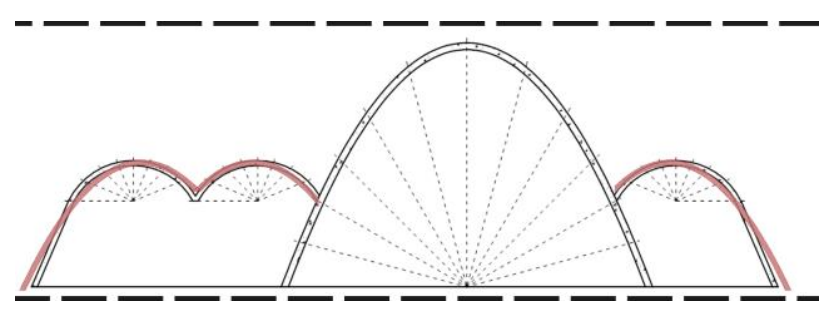

Figura 10 - Etapa ACG. Fonte: autores.

Adicionalmente, com o propósito de checar como seria a forma proposta por Niemeyer caso considerasse apenas as parábolas, a modelagem paramétrica foi materializada em escala reduzida por meio da prototipagem rápida dos elementos matematizados. Nela, foi possível observar sensorialmente, por meio do toque na superfície do protótipo, as camadas de impressão contendo diversas parábolas interpoladas (Figura 11).

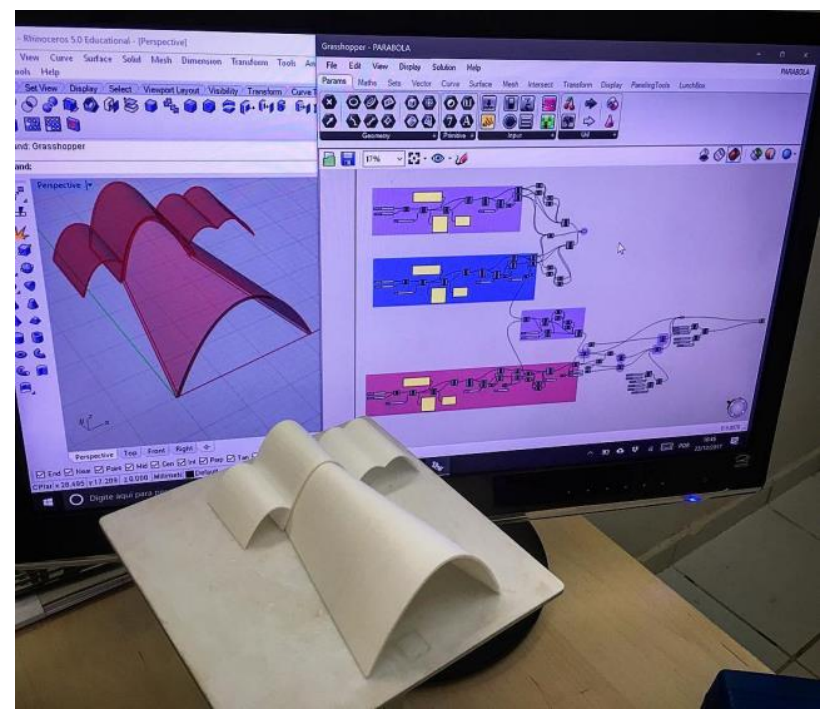

Figura 11 - Modelagem paramétrica e protótipo das formas parabólicas da Pampulha. Fonte: autores.

\section{RESULTADOS E DISCUSSÃO}

É comum na arquitetura de Oscar Niemeyer projetos onde a forma construída apresenta uma geometria simples e o espaço arquitetônico exibe diversas contrações e dilatações, enriquecendo o caminhar pelo espaço projetado pelo arquiteto (Florio \& Veiga, 2016), e na igreja da Pampulha não é diferente.

A inserção dos dados matemáticos nos algoritmos e a posterior aplicação Operações Geométricas Euclidianas e Booleanas possibilitaram a construção do modelo paramétrico e a conseguinte materialização com a prototipagem rápida.

A partir da sobreposição da etapa ACG, observamos que as supostas parábolas 3, 4 e 5 não são de fato parábolas, pois possuem propriedade geométrica que aparentam ter 
a forma simplificada de arcos e linhas que concordam entre si. Caso fossem de fato parábolas, deveriam ter aberturas laterais maiores e a parte superior da curva, que faz o papel de cobertura, seria mais comprimida.

Durante a análise do modelo geométrico final, foi detectada geometria distinta de uma curva cônica na execução das parábolas de 3 a 5 , que podem ter sido obtidas por meio de concordância entre linhas oblíquas e arcos. Pressupomos que essa decisão de racionalizar a geometria pode ter sido motivada pela necessidade de otimizar o processo de concretagem, pois duas retas unidas por um arco concordante podem produzir bem menos cortes de fôrma (que emoldurou o concreto no local da obra) do que o formato parabólico.

A utilização da modelagem paramétrica pode ser uma ferramenta de análise voltada não apenas a projetos contemporâneos, mas a ao estudo de obras já existentes. Assim, utilizando algoritmos aplicados a gráfica digital, aliados a conhecimentos matemáticos, arquitetos e engenheiros calculistas podem ter melhor compreensão geométrica de obras arquitetônicas importantes que fugiram à convencionalidade da sua época.

\section{AGRADECIMENTOS}

Agradecemos ao campus São Gonçalo do Amarante, Instituto Federal do Instituto Federal do Rio Grande do Norte, pelo apoio na aquisição de equipamentos para a pesquisa.

\section{REFERÊNCIAS}

Burry, J., \& Burry, M. (2010). The New Mathematics of Architecture $\left(1^{\mathrm{a}}\right)$. London: Thames \& Hudson.

Florio, W., \& Veiga, B. T. M. da. (2016). A visualização espacial através da modelagem paramétrica e da fabricação digital em edifícios curvilíneos de Oscar Niemeyer no Memorial da América Latina The spatial visualization through parametric modeling and digital fabrication in curvilinear building. In SIGraDi 2016, XX Congress of the Iberoamerican Society of Digital Graphics (pp. 686-692).

Gonçalves, S. N. L. (2010). Museus projetados por Oscar Niemeyer de 1951 a 2006: o programa como coadjuvante. Universidade de São Paulo.

lezzi, G. (1993). Fundamentos de matemática elementar, 7 (4ª). São Paulo: Editora Atual.

Kolarevic, B. (2008). The (risky) craft of digital making. In Manufacturing Material Effects: Rethinking Design and Making in Architecture. New York: Routledge.

Lima, E., Carvalho, P. C. P., Morgado, A. C., \& Wagner, E. (2007). A Matemática do Ensino Médio - Volume $3\left(6^{a}\right)$. Rio de Janeiro: SBM.

Pereira, N. C. (2012). Conceoção arquitetônica e estrutural de duas obras de Oscar Niemeyer: igreja da Pampulha e Pavilhão da Gameleira. Universidade de Brasília.

Shekhawat, K. (2017). Mathematical propositions associated with the connectivity of architectural designs. Ain Shams Engineering Journal, 8(4), 653-661. https://doi.org/10.1016/J.ASEJ.2015.09.009

Souza, M. H. (2012). Clássicos da Arquitetura: Igreja da Pampulha / Oscar Niemeyer | ArchDaily Brasil. Retrieved June 16, 2018, from https://www.archdaily.com.br/br/0183469/classicos-da-arquitetura-igreja-da-pampulha-slashoscar-niemeyer

Stals, A., Elsen, C., \& Jancart, S. (2017). Practical Trajectories of Parametric Tools in Small and Medium Architectural Firms. CAAD Futures, 461-473.

Tedeschi, A. (2014). AAD_Algorithms Aided Design: Parametric Strategies Using Grasshopper. Brienza Potenza: Le Penseur Publisher.

Woodbury, R. (2010). Elements of Parametric Design. New Yoek: Routledge. 